\title{
Studies on Quark Confinement in a Proton on the Basis of Interaction Potential
}

\author{
Shinichi Ishiguri \\ Nihon University \\ 1-2-1 Izumi-Cho, Narashinoshi, Chiba 275-8575 JAPAN \\ TEL: +81-47-474-9143 \\ Email: ishiguri.shinichi@nihon-u.ac.jp
}

\begin{abstract}
This study describes quark confinement in terms of linear interaction potentials. The three quarks in a proton are assumed to revolve around a common center and have masses determined as if they were Dirac particles. Under these assumptions, the magnetic moment of a proton is derived via Maxwell's equations. Moreover, the rotational motion of the quarks can be thought of as an electrical current that induces a magnetic field. Thus, the scalar product of the magnetic moment and the magnetic field describes a linear interaction potential between the quarks that gives the mass of the proton. The proton mass as predicted by this physical model is in good agreement with experimental observations and requires no numerical calculations. Thus, the simple physical model suggests a solution for the problem of quark confinement by modeling the strong force as an interaction potential.
\end{abstract}

Keywords: quark, linear interactive potential, mass of a proton

\section{Introduction}

Hadrons and quarks have been studied for about half a century. A proton is composed of three quarks [1-7]. The proton mass has been measured with good accuracy, but cannot be predicted theoretically using analytic solutions. The required numerical calculations to determine the proton mass are well known in the field of quantum chromodynamics (QCD), which can calculate the mass of hadrons using a supercomputer [8-12]. Moreover, the QCD model is described by the SU(3) and Yang-Mills fields [13-15].

However, the calculation time needed is extremely long even with supercomputers, and the color charges employed by QCD are controversial because the color charge of the gluons that mediate the strong interaction has not yet been measured. Considering these facts, we claim that QCD is not inadequate but insufficient, and the quark-confinement problem, or the question of why the proton persists, remains unsolved because the numerical calculations involved in lattice QCD implies so- 
called an ideal experiment, which does not teach us why the quark is not measured singly and why the proton is not destroyed permanently. Although the lattice QCD calculations for the mass of hadrons have been conducted, based on experimental observations of $\Omega$ particles i.e., the parameters were determined from known experimental values such that the numerical calculations predict the masses of other unobserved hadrons [16]. This result is meaningful to some extent, but the calculations cannot stand independently without numerical modeling. Thus, these results also fail to explain the basic physical picture of quark confinement.

In this paper, we use more simple and basic calculations than the QCD approach with color charges to calculate the mass of a proton. This physical model yields the linear interaction potential between quarks, the magnetic moment, and the mass of a proton without numerical calculations. This paper assumes that three quarks have rotational moments and the quark mass is determined from the energy gap. The quarks' rotational motion is thought of as an electrical current that induces a magnetic field, which explains the proton's magnetic moment. The scalar product of the magnetic moment and the magnetic field produces the linear interaction potential between quarks that predicts the mass of a proton.

When comparing these analytic results with experimental observations, the analytic model corresponds well with the observed mass of a proton. The derived linear potential provides a mechanism that explains how a larger relative distance between quarks causes a stronger attractive force between them. This fact implies that quarks cannot be measured in isolation. Although several articles have modeled quark confinement numerically $[12,13]$, the present paper employs analytic expressions without numerical methods, thus giving a purely physical model for quark confinement.

\section{Theory}

As shown in Fig. 1, circular coordinates with radius $r$ are considered. Each apex has a quark whose electric charge is $1 / 3 q$. In this model, each quark revolves around the origin $\mathrm{O}$, maintaining a constant relative distance between the three quarks. Fig. 1 therefore gives a simple model of a proton.

The rotational motion of the three quarks can be modeled as the current $I$ if we think of the quarks as charged particles,

$I=\frac{3 \times(1 / 3) q}{T}=\frac{\omega q}{2 \pi},(1)$

where $T, q$, and $\omega$ denote the period of the rotation movement, the charge of an electron, and the angular frequency of the motion, respectively.

A magnetic moment is generally defined as

$\mathrm{P}=\mathrm{IS},(2)$

where $\mathrm{P}, \mathrm{I}$, and $\mathrm{S}$ denote the magnetic moment, the current, and the area, respectively.

The area $\mathrm{S}$ is simply 
$\mathrm{S}=\pi r^{2}$

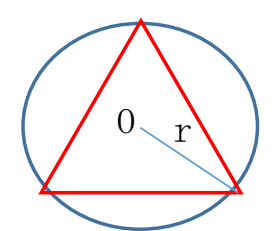

Fig. 1 a model of a proton. Three quarks are located at each apex of equilateral triangle. Maintaining the shape of the triangle, the rotational motion along the origin $\mathrm{O}$ is introduced

The following assumptions are introduced.

1) The quark mass is determined by the energy gap of the vacuum (i.e., it is a Dirac particle)

2) The rotational angular frequency is determined by the energy gap from the quarks' vacuum.

3) This Dirac particle is described as

$$
\frac{1}{2} \hbar \omega=3 m_{A} c^{2},
$$

where the left-hand side gives the zero-point energy of the quark in a vacuum and the right-hand side is the sum of the three quarks' energy at rest, with $m_{A}$ being the mass of a single quark.

Note that, in a vacuum, the following equation holds for an electron:

$$
\frac{1}{2} \hbar \omega=m_{e} c^{2},
$$

where $m_{\mathrm{e}}$ denotes the mass of the electron.

Considering the above, the magnetic moment of a proton $p$ is

$p=\frac{q}{2 \pi} \frac{6 m_{A} c^{2}}{\hbar} \pi r^{2}$.

The magnetic field $H$ induced by current $I$ is

$\mathrm{H}=\frac{I}{2 r}=\frac{\omega q}{4 \pi r}=\frac{q}{4 \pi r} \frac{6 m_{A} c^{2}}{\hbar}$.

Thus, in combination with the magnetic moment $p$, the linear interaction potential results:

$\mathrm{E}=-\mathrm{p} \cdot \mathrm{H}=-q^{2} \frac{1}{8 \pi}\left(\frac{6 m_{A} c^{2}}{\hbar}\right)^{2} r$,

This potential yields the proton mass:

$|E|=m_{p} c^{2}$, 
where, $m_{\mathrm{p}}$ denotes the proton mass.

\section{Result and Discussion}

This section compares experimental observations with the value of the proton mass calculated using the model described above.

The physical parameters listed in Table 1 were used for the calculations.

Table 1 parameters for hand calculations

\begin{tabular}{ll}
\hline Plank constant $\hbar$ & $1.05 \times 10^{-34} \mathrm{Js}$ \\
Electric charge $\mathrm{q}$ & $1.6 \times 10^{-19} \mathrm{C}$ \\
Quark mass $\mathrm{m}_{\mathrm{A}}$ & $3 \times 9.1 \times 10^{-31} \mathrm{Kg}$ \\
Proton radius $\mathrm{r}$ & $0.8 \times 10^{-15} \mathrm{~m}$
\end{tabular}

The results are listed in Table 2. Experimental data are averaged from two sources [15,17]. As shown, the theory of this paper well predicts the mass of a proton.

Table 2 comparison of the mass of a proton

$\begin{array}{ll}\text { Hand calculation } & 1.8 \times 10^{-27} \mathrm{Kg} \\ \text { Experiment } & 1.67 \times 10^{-27} \mathrm{Kg}\end{array}$

Typically, strong-force interactions are calculated using lattice QCD. However, this calculation using QCD requires numerical methods, so the resulting values do not have the picture sufficiently, which describes why the quark is not measured singly and why the proton is not destroyed permanently. Here, we started with a simple physical model of three quarks forming an equilateral triangle, and used basic physical relations to derive the magnetic moment and mass of the proton. In the process, we derived the linear interaction potential. Thus, the interaction potential increases along with the relative distance between quarks. This relation explains why protons persist and why single quarks have not been observed. Thus, the mass of the proton is explained by the model above.

The physical model above conceives of quarks revolving around a common center and having mass correlated with the energy gap in a vacuum. The rotational motion explains the magnetic moment of the proton. Moreover, because the quarks' rotational motion induces a current and magnetic field, the scalar product of the magnetic field and the magnetic moment produces a linear interaction potential between the quarks that explains the mass of the proton. Note that the derived magnetic moment is 
combined with the magnetic field via the scalar product, as indicated in eq. (8). This implies that the single magnetic moment $p$ is independent of the generally measured magnetic moment, i.e., the magnetic moment $p$ cannot be observed in isolation. Therefore, the generally measured magnetic moment must be considered separately for that of the present paper.

\section{Conclusion}

This paper has formulated the problem of quark confinement in a novel manner.

Three quarks revolve around a common center and have mass. This rotational motion produces a current and the magnetic field, from which the magnetic moment of the proton can be calculated. Moreover, following Maxwell's laws, the scalar product of the magnetic moment and the magnetic field results in a linear potential between the quarks that yields the mass of the proton. This derived linear potential between the quarks explains the problem of quark confinement, since the potential increases along with the distance between quarks. This relation between the quark spacing and the linear potential explains why quarks are never observed in isolation, and why the proton coheres.

In future work, the same methods will be used to predict the mass of a meson.

\section{References}

1) M. Gell-Mann, Physics letters 8 (3) 214-215 (1964)

2) G. Zweig, CERN Report, No 8182/TH.401

3) J. Gasser, et al., Physics Report 87 (3) 77- 169 (1982)

4) A. D. Rujula, et al., Physical Review D 12, 147 (1975)

5) D. Gross, F. Wilczek, Physics Review 30, 1343-1346 (1973)

6) H.D. Politzer, Physical Review Letters 30, 1346-1349 (1973)

7) T. Gutsche, et al., Physical Review D 94, 112030 (2016)

8) M. Bander, Physics Reports 75 (4) 205-286 (1981)

9) Z. G. Tan, et al., Results in Physics 12, 190-192 (2019)

10) A. M. Mack, Physics Letters B 78 (2-3), 263-268 (1978)

11) K. G. Wilson, Physical Review D 10 (8) 2445-2459 (1974)

12) M.E. Carrillo-Serrano, Physics Letters B, 759, 178-183 (2016)

13) M. Y. Han, Y. Nambu, Physical Review 139, B1006 (1995)

14) Yang, C. N., Mills, R., Physical Review 96 (1) 191-195 (1954)

15) R. Sakata, Introduction to Condensed Matter Physics, Bayhukan (1989)

16) Y. Kakiba, "Physics of Quark Gluon Plasma-Recreating Hot Matter in Early Universe in Laboratory", Kyouritsu shuppan, p. 62 (2014)

17) V. Khachatryan et al., Physical Review D 93, 072004 (2016) 


\section{Acknowledge}

We thank the Enago for the English review.

\section{Additional information}

This paper is not related to any competing interests such as funding, employment and personal financial interesting. Moreover, this paper is not related to non-financial competing interesting 